\title{
Evaluation of connectivity, flux pinning, and upper critical field contributions to the critical current density of bulk pure and SiC-alloyed $\mathrm{MgB}_{2}$
}

\author{
A. Matsumoto, $\left.{ }^{a}\right) \mathrm{H}$. Kumakura, and H. Kitaguchi \\ National Institute for Materials Science, 1-2-1 Sengen, Tsukuba 305-0047, Japan \\ B. J. Senkowicz, M. C. Jewell, E. E. Hellstrom, Y. Zhu, P. M. Voyles, and D. C. Larbalestier \\ Applied Superconductivity Center, University of Wisconsin-Madison, Madison, Wisconsin 53706
}

(Received 1 February 2006; accepted 3 August 2006; published online 28 September 2006)

\begin{abstract}
Measurement of critical current density $J_{c}$, normal state resistivity $\rho_{n}$, and upper critical field $H_{c 2}$ on pure and $10 \%$ SiC-doped $\mathrm{MgB}_{2}$ bulks show systematic enhancement of $H_{c 2}$ by $\mathrm{SiC}$ addition and by lowering reaction temperature. $H_{c 2}(10 \mathrm{~K})$ exceeds $33 \mathrm{~T}$, while the extrapolated zero temperature value exceeds $40 \mathrm{~T}$. The Rowell [Supercond. Sci. Technol. 16, R17 (2003)] analysis suggests that only $8 \%-17 \%$ of the $\mathrm{MgB}_{2}$ cross section actually carries current. Higher reaction temperature enhances the connectivity but degrades $H_{c 2}$ and flux pinning, making the measured $J_{c}$ a complex balance between connectivity, $H_{c 2}$, and flux pinning induced by grain boundaries and precipitates. (c) 2006 American Institute of Physics. [DOI: 10.1063/1.2357027]
\end{abstract}

Magnesium diboride ${ }^{1}\left(\mathrm{MgB}_{2}\right)$ is a potential competitor to $\mathrm{Nb}$-base superconductors due to its lower raw material cost and good $H_{c 2}$ values. However, some confusion often surrounds the meaning of measured $H_{c 2}$ values in untextured samples, which are almost always the higher value $H_{c 2}^{\|}$, where the applied field $H$ is parallel to the $a b$ planes. Sometimes the irreversibility field $H_{\text {irr }}$ is defined by the bottom of this $H_{c 2}^{\|}$transition, making $\mathrm{MgB}_{2}$ look very good in comparison to an isotropic superconductor such as $\mathrm{Nb}_{3} \mathrm{Sn}$. But the real measure should be the $H_{\text {irr }}$ defined by the extrapolation of the critical current density $J_{c}(H)$ to zero, which is typically only about half of $H_{c 2}^{\|}$, because $H_{\text {irr }}$ is determined by the breadth of the lower, perpendicular $H_{c 2}$ transition, $H_{c 2}^{\perp}$. At $4.2 \mathrm{~K}$, measured $H_{c 2}^{\|}$values ${ }^{2-7}$ now are very similar to those of $\mathrm{Nb}_{3} \mathrm{Sn}, \sim 25-27 \mathrm{~T},{ }^{8}$ but $H_{\text {irr }}$ values are only $12-17 \mathrm{~T}$ due to suppression of current flow in those grains oriented with $\mathrm{Mg}$ and $\mathrm{B}$ planes perpendicular to the applied field, which explains why defining $H_{\text {irr }}$ by the bottom of the $H_{c 2}^{\perp}$ transition is more appropriate for untextured samples than the bottom or $10 \%$ point on the small-current-density, resistive $H_{c 2}$ transition which corresponds to $H_{c 2}^{\|}$. However, the resistive $H_{c 2}$ transition is still useful for measuring the breadth of the parallel $H_{c 2}$ transition $\Delta H$, which may be indicative of inhomogeneity in composition in the sample. Hopes for expanding the useful range of $\mathrm{MgB}_{2}$ are encouraged by earlier work that has shown that $H_{c 2}^{\|}(0)$ can exceed $70 \mathrm{~T}$ in C-doped $\mathrm{MgB}_{2}$ thin films, ${ }^{2}$ but so far the highest $H_{c 2}(0)$ of $\mathrm{C}$ - or SiC-doped wires or bulks is $\sim 35 \mathrm{~T},{ }^{3,4,9,10}$ only half this value. Since $H_{c 2}$ and $H_{\text {irr }}$ enhancement is crucial for magnet applications, we have here systematically studied the $H_{c 2}$ transition and $J_{c}(H, T)$ behavior of pure and $\mathrm{SiC}$-doped bulks. Irrespective of this high-field perspective on $\mathrm{MgB}_{2}$, we should also point out that $J_{c}(H)$ falls off only slowly in the $10-30 \mathrm{~K}$ range, making $\mathrm{MgB}_{2}$ useful for lower field applications without liquid He.

Our previous reports ${ }^{6,7}$ showed that higher $J_{c}$ values were obtained in tapes using $\mathrm{MgH}_{2}$ rather than $\mathrm{Mg}$ powder.

\footnotetext{
${ }^{\text {a) }}$ Author to whom correspondence should be addressed; electronic mail: matsumoto.akiyoshi@nims.go.jp
}

Nano-SiC addition improved the high-field $J_{c}$ at low temperatures and produced a measured $H_{c 2}$ value of $23 \mathrm{~T}$ at 4.2 K. Here we present a more detailed study of $\mathrm{MgB}_{2}$ samples cut from this same tape measured without any extraneous sheath material.

$\mathrm{MgB}_{2}$ bulk samples were prepared by conventional in situ powder-in-tube method with commercial $\mathrm{MgH}_{2}$ and amorphous B powders which were mixed and packed into a pure Fe tube in air. 5 or $10 \mathrm{~mol} \%$ of $\sim 30 \mathrm{~nm} \mathrm{SiC} \mathrm{powder}{ }^{5}$ was added for the doped samples. The filled tubes were groove rolled into $2 \mathrm{~mm}$ square rods and then flat rolled into $0.5 \mathrm{~mm}$ thick by $4 \mathrm{~mm}$ wide tapes. $50 \mathrm{~mm}$ long samples were heat treated at $600,700,800$, and $900{ }^{\circ} \mathrm{C}$ for $1 \mathrm{~h}$ under $\mathrm{Ar}$ atmosphere making the 12 -sample set. ${ }^{7}$ After peeling away the Fe sheath, resistivity curves were measured with $5 \mathrm{~mA}$ transport currents in a $9 \mathrm{~T}$ Quantum Design physical properties measurement system, the $33 \mathrm{~T}$ Bitter magnet at the National High Magnetic Field Laboratory (NHMFL) in Tallahassee, and the $60 \mathrm{~T}$ short pulse magnet at the NHMFL in Los Alamos National Laboratory. The $10 \%$ and $90 \%$ points on the resistive transition curves were used to define a transition breadth $\Delta H$ and $H_{c 2}^{\|}$. Magnetization properties were measured in an Oxford Instruments vibrating sample magnetometer, from which the critical current density $J_{c}(H, T)$ was calculated assuming fully connected samples using the expression $J_{c}(H, T)=0.5 \Delta M 12 b /\left(3 b d-d^{2}\right)$, where $b$ and $d$ are the width and thickness of the rectangular section bar. Extrapolation of $J_{c}(H)$ to zero allowed extraction of $H_{\text {irr }}$. However, following Rowell, ${ }^{11}$ we believe that the connected cross section $1 / F$ of our samples is much less than unity, based on calculations of $1 / F$ using the relation $\rho(T)$ $=F\left[\Delta \rho_{\mathrm{sc}}(T)+\rho(0)\right]$, where $\rho_{n}$ is the measured normal state resistivity and $\Delta \rho_{\mathrm{sc}}(300-50 \mathrm{~K})=7.3 \mu \Omega \mathrm{cm}$ (Table I).

Table I provides an overview of the properties of the four samples. $\mathrm{SiC}$ additions depress $T_{c}$, raise $H_{c 2}$, broaden the $H_{c 2}$ transition, and raise $\rho_{n} .1 / F$ factors are variable and much less than $100 \%$, implying that the variation in measured $\rho_{n}$ in Table I is only partly due to scattering. The best connected sample was $900{ }^{\circ} \mathrm{C}$ pure at only $17 \%$, while the two $600{ }^{\circ} \mathrm{C}$ samples were only $8 \%-9 \%$ connected. Reaction temperature also played a large role: $T_{c}$ was lower, $\rho_{n}$ higher, 
TABLE I. Superconducting properties of the $\mathrm{MgB}_{2}$ samples as a function of reaction temperature and $\mathrm{SiC}$ addition.

\begin{tabular}{|c|c|c|c|c|c|c|c|c|}
\hline Sample & $\begin{array}{c}T_{c}^{\text {zero }} \\
(\mathrm{K})\end{array}$ & $\begin{array}{l}\rho_{n}(40 \mathrm{~K}) \\
(\mu \Omega \mathrm{cm})\end{array}$ & RRR & $\begin{array}{l}\text { Active cross } \\
\text { section }(\%)\end{array}$ & $\begin{array}{l}J_{c}(5 \mathrm{~T}, 4.2 \mathrm{~K}) \\
\left(\times 10^{4} \mathrm{~A} / \mathrm{cm}^{2}\right)\end{array}$ & $\begin{array}{c}J_{c}(1 \mathrm{~T}, 25 \mathrm{~K}) \\
\left(\times 10^{4} \mathrm{~A} / \mathrm{cm}^{2}\right)\end{array}$ & $\begin{array}{l}\Delta H(10 \mathrm{~K}) \\
\quad(\mathrm{T})\end{array}$ & $\begin{array}{c}H_{c 2}(10 \mathrm{~K}) \\
(\mathrm{T})\end{array}$ \\
\hline $600{ }^{\circ} \mathrm{C}$ pure & 36.0 & 116 & 1.70 & 9.0 & 3.5 & 4.2 & 6.3 & 24.7 \\
\hline $900{ }^{\circ} \mathrm{C}$ pure & 37.5 & 31 & 2.40 & 16.6 & 12 & 4.9 & 3.5 & 17.2 \\
\hline $600{ }^{\circ} \mathrm{C} 10 \% \mathrm{SiC}$ & 32.0 & 324 & 1.27 & 8.2 & 3.3 & 0.6 & 10.2 & 31.6 \\
\hline $900^{\circ} \mathrm{C} 10 \% \mathrm{SiC}$ & 35.5 & 100 & 1.58 & 12.7 & 7.9 & 5.3 & 3.8 & 26.7 \\
\hline
\end{tabular}

$H_{c 2}^{\|}$higher, and $H_{c 2}$ transition broader for the $600{ }^{\circ} \mathrm{C}$ reaction.

Figure 1 shows $J_{c}(H)$ measured at 4.2 and $25 \mathrm{~K}$ but the values are not easy to systematize because of the competing influences of connectivity, $H_{c 2}$, and flux pinning. At $H$ $>6 \mathrm{~T}$, the $\mathrm{SiC}$-doped samples have higher $J_{c}(4.2 \mathrm{~K})$ than the pure samples, consistent with their higher $H_{c 2}$ (see Fig. 4). At low $H$ where $H_{c 2}$ is not important, $J_{c}(4.2 \mathrm{~K})$ for the $600{ }^{\circ} \mathrm{C}$-SiC sample drops below the $900{ }^{\circ} \mathrm{C}$-SiC sample, consistent with its smaller connectivity (8\% vs $12 \%)$. By contrast, $J_{c}(4.2 \mathrm{~K})$ of the pure samples is higher for the $600{ }^{\circ} \mathrm{C}$ reaction than for the $900{ }^{\circ} \mathrm{C}$ reaction for all $H$, a striking contrast with the expectation from the approximate halving of connectivity (9\% vs $17 \%$ ) seen in Table I. However, transmission electron microscopy (TEM) analysis ${ }^{12}$ shows that the $\mathrm{MgB}_{2}$ grain size is significantly smaller after $600{ }^{\circ} \mathrm{C}$ rather than after $900{ }^{\circ} \mathrm{C}$ reaction (Fig. 2). Consistent with $\mathrm{Nb}_{3} \mathrm{Sn}$ where fine grain pinning dominates, ${ }^{13}$ the pure $\mathrm{MgB}_{2}$ samples show a flux-line lattice shear shape of $F_{p}$ curve. The high $J_{c}$ data of the very fine grain pure $600{ }^{\circ} \mathrm{C}$ reaction are consistent with strong vortex pinning by grain boundaries, which have been shown ${ }^{14}$ to be an important pinning center in $\mathrm{MgB}_{2}$. However, the low connectivities in Table I also suggest that the local, vortex pinning $J_{c}$ of our samples is up to an order of magnitude higher than indicated in Fig. 1. Finally we note that $J_{c}(H, 25 \mathrm{~K})$ shows significant effects from $T_{c}$. For example, the $600{ }^{\circ} \mathrm{C}$-SiC sample has the lowest $J_{c}(H)$ and the lowest $T_{c}(32 \mathrm{~K})$.

Figure 3 shows the normalized pinning force at $25 \mathrm{~K}$ of the four samples. The SiC-doped samples show higher vortex pinning strength at higher magnetic fields than the pure samples. Microstructural analysis ${ }^{12}$ shows that the $\mathrm{SiC}$ samples contain a significant density of various nanoscale precipitates, consistent with this additional pinning. The pinning force $F_{p}$ curve shapes were rather independent of temperature.

The full range of $H_{c 2}^{\|}$data is not easy to show in one plot due to the breadth and overlap of the data sets, so in Fig. 4 we show the range $\Delta H$ between the $10 \%$ and $90 \%$ points on the $H_{c 2}$ transition as a function of measurement temperature only for the $900{ }^{\circ} \mathrm{C}$-pure and $600{ }^{\circ} \mathrm{C}$-SiC samples, which have the narrowest $(4.5 \mathrm{~T}$ at $2 \mathrm{~K})$ and the broadest $(18 \mathrm{~T}$ at $2 \mathrm{~K}$ ) transitions of our sample set. The $600{ }^{\circ} \mathrm{C}$-SiC sample has $H_{c 2}(0 \mathrm{~K})=42 \mathrm{~T}$, the highest in our set, considerably higher than in C-doped single crystals ${ }^{10}$ or filaments, ${ }^{9}$ and exceeded only by some thin films. ${ }^{4}$

It is becoming increasingly clear that most $\mathrm{MgB}_{2}$ bulk samples have rather inhomogeneous electromagnetic properties. High porosity associated with the $\sim 40 \%$ volume contraction of the reaction $\mathrm{MgH}_{2}+\mathrm{B}=\mathrm{MgB}_{2}$ provides part of the reason for the low connectivity shown in Table I but our microstructural studies $^{12}$ show that various grain boundary phases are present in both pure and $\mathrm{SiC}$-doped samples. Both Downloaded 29 Oct 2006 to 128.104.1.219. Redistribution subject

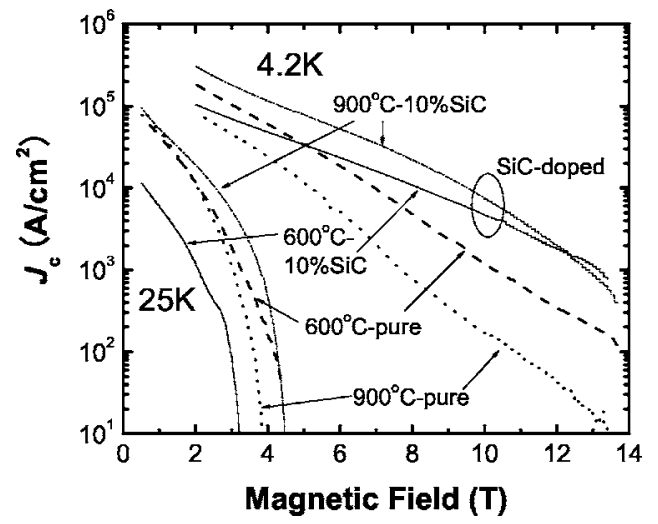

FIG. 1. $J_{c}-H$ curves at 4.2 and $25 \mathrm{~K}$ derived from magnetization measurements.

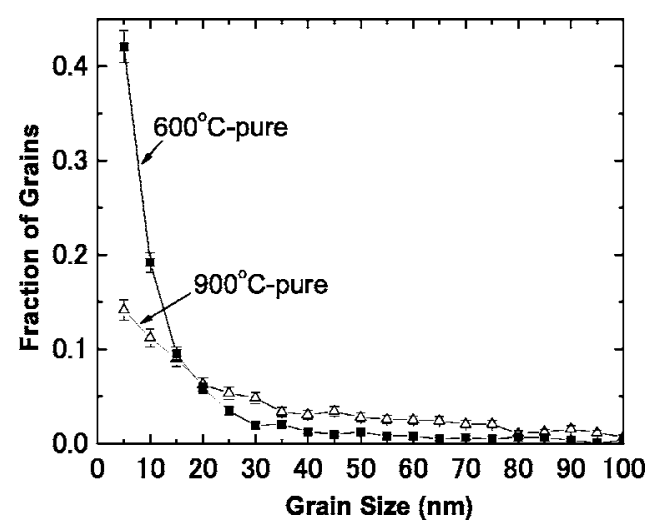

FIG. 2. Grain size distributions for the two pure samples, measured from TEM dark field images. Both samples show a preponderance of grains $<50 \mathrm{~nm}$ in diameter, but grain growth does occur at $900{ }^{\circ} \mathrm{C}$. Doped samples show the same trend, but analysis is complicated by $\mathrm{SiC}$ reaction phases. Approximately $7 \%$ of the grains in the $600{ }^{\circ} \mathrm{C}$ sample and $9 \%$ of the grains in the $900{ }^{\circ} \mathrm{C}$ sample are larger than $100 \mathrm{~nm}$ and are not included in the figure.

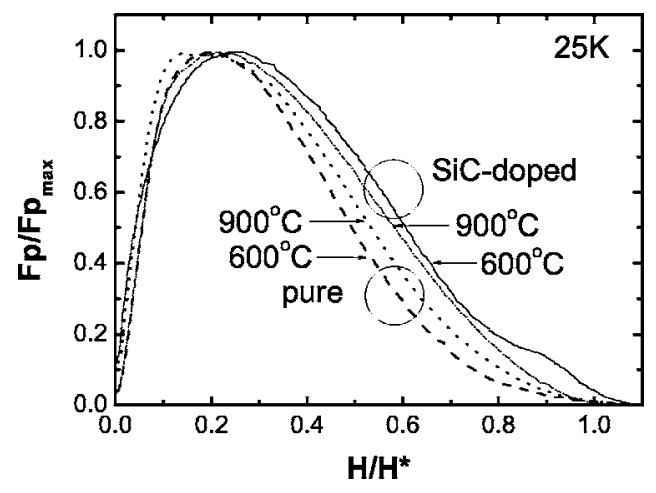

FIG. 3. Normalized pinning force of the four samples at $25 \mathrm{~K}$, where the criterion for the irreversibility field is $J_{c}=100 \mathrm{~A} / \mathrm{cm}^{2}$. The SiC-doped samples show distinct additional pinning in higher reduced fields, a result seen at all temperatures. 


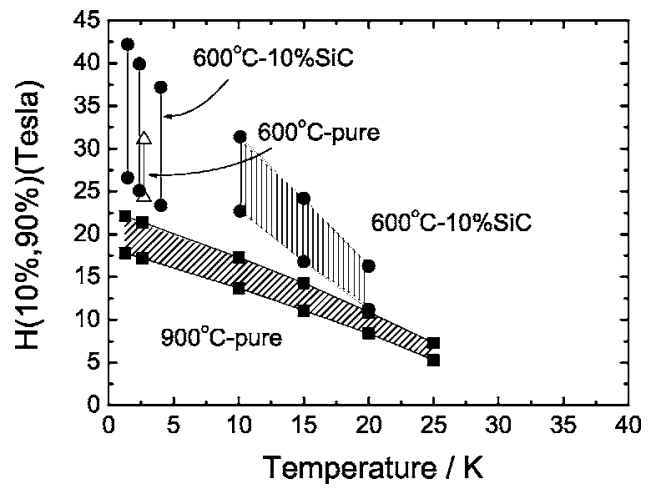

FIG. 4. $90 \%$ and $10 \%$ of normal state resistivities in the resistive transition curves for two samples of $\mathrm{MgB}_{2} .90 \%$ of normal state resistivity is taken as $H_{c 2}$. The much broader transition of $\Delta H(90 \%-10 \%)$ of the $600{ }^{\circ} \mathrm{C}-\mathrm{SiC}$ sample suggests that significant inhomogeneity is introduced by alloying and by reaction at low temperatures. This sample also has the largest upper critical field.

factors may play a significant role in producing connectivity factors that lie only between $8 \%$ and $17 \%$. Partially offsetting these low connectivities is the observation of very fine grains of $<50 \mathrm{~nm}$ in all four samples, which appear to produce very strong flux pinning. The most frequently observed grain size in the $600{ }^{\circ} \mathrm{C}$ samples is $<20 \mathrm{~nm}$, a very favorable size compared to the $\sim 150 \mathrm{~nm}$ grain size seen in optimum, high- $J_{c}$, high- $H_{c 2} \mathrm{Nb}_{3} \mathrm{Sn}^{8}{ }^{8}$

In summary our study shows that very high values of $H_{c 2}(0)$ exceeding $40 \mathrm{~T}$ can be attained in SiC-doped bulk $\mathrm{MgB}_{2}$ but also that extended transitions with considerable smearing exist in the higher $H_{c 2}$ samples. Strong vortex pinning is indicated both by $J_{c}$ up to $10^{4} \mathrm{~A} / \mathrm{cm}^{2}$ at $10 \mathrm{~T}, 4.2 \mathrm{~K}$ and by distortion of the pinning force curve at high reduced fields in SiC-doped samples.
This research was partially funded by NSF through the MRSEC and the Focused Research Group at UW-Madison. One of the authors (A.M.) was supported by the Ministry of Education, Culture, Sports, Science and Technology in Japan, while another author (B.J.S.) was supported by the Fusion Energy Sciences Fellowship Program administered by Oak Ridge Institute for Science and Education under contract between the U.S. DOE and the Oak Ridge Associated Universities.

${ }^{1}$ J. Nagamatsu, N. Nakagawa, T. Muranaka, Y. Zenitani, and J. Akimitsu, Nature (London) 410, 63 (2001).

${ }^{2}$ C. B. Eom, M. K. Lee, J. H. Choi, L. J. Belenky, X. Song, L. D. Cooley, M. T. Naus, S. Patnaik, J. Jiang, M. Rikel, A. Polyanski, A. Gurevich, X. Y. Cai, S. D. Bu, S. E. Babcock, E. E. Hellstrom, D. C. Larbalestier, N. Rogado, K. A. Regan, M. A. Hayward, T. He, J. S. Slusky, K. Inumaru, M. K. Haas, and R. J. Cava, Nature (London) 411, 558 (2001).

${ }^{3}$ V. Braccini, L. D. Cooley, S. Patnaik, D. C. Larbalestier, P. Manfrinetti, A. Palenzona, and A. S. Siri, Appl. Phys. Lett. 81, 4577 (2002).

${ }^{4}$ B. J. Senkowicz, J. E. Gienke, S. Patnaik, C. B. Eom, E. E. Hellstrom, and D. C. Larbalestier, Appl. Phys. Lett. 86 (2005).

${ }^{5}$ S. X. Dou, S. Soltanian, J. Horvat, X. L. Wang, S. H. Zhou, and M. Ionescu, Appl. Phys. Lett. 82, 4996 (2003).

${ }^{6}$ H. Kumakura, H. Kitaguchi, A. Matsumoto, and H. Hatakeyama, Appl. Phys. Lett. 84, 3669 (2004).

${ }^{7}$ A. Matsumoto, H. Kumakura, H. Kitaguchi, and H. Hatakeyama, Supercond. Sci. Technol. 16, 926 (2003).

${ }^{8}$ A. Godeke, M. C. Jewell, C. M. Fischer, A. A. Squitieri, P. J. Lee, and D. C. Larbalestier, J. Appl. Phys. 97, 093909 (2005).

${ }^{9}$ R. H. T. Wilke, S. L. Bud'ko, P. C. Canfield, D. K. Finnemore, Raymond J. Suplinskas, and S. T. Hannahs, Phys. Rev. Lett. 92, 217003 (2004).

${ }^{10}$ S. M. Kazakov, R. Puzniak, K. Rogacki, A. V. Mironov, N. D. Zhigadlo, J. Jun, Ch. Soltmann, B. Batlogg, and J. Karpinski, Phys. Rev. B 71, 024533 (2005).

${ }^{11}$ J. M. Rowell, Supercond. Sci. Technol. 16, R17 (2003).

${ }^{12}$ Y. Zhu, A. Matsumoto, H. Kumakura, H. Kitaguchi, B. J. Senkowicz, M. C. Jewell, E. E. Hellstrom, D. C. Larbalestier, and P. M. Voyles, J. Appl. Phys. (to be published).

${ }^{13}$ J. Kramer, J. Appl. Phys. 44, 1360 (1973).

${ }^{14}$ H. Kitaguchi, A. Matsumoto, H. Kumakura, T. Doi, H. Sosiati, and S. Hata, Appl. Phys. Lett. 85, 2842 (2004). 